\title{
ANTIMICROBIAL ACTIVITY OF SOME BIOCIDES AGAINST MICROORGANISMS ISOLATED FROM A SHARED STUDENT KITCHEN
}

\author{
Karim Hassan $^{1 *}$ and Marwan El Bagoury ${ }^{2}$ \\ ${ }^{1}$ School of Pharmacy and Pharmaceutical Sciences, The University of Manchester, UK \\ ${ }^{2}$ Universität Ulm - Medizinische Fakultät, Ulm, Deutschland \\ *E-mail: karimhassan66@ outlook.com
}

\begin{abstract}
Effective biocide use in the kitchen is essential in order to minimize the risk of infection, especially in shared kitchens. The study aimed to assess the susceptibility of microorganisms previously isolated from a shared student kitchen to common kitchen biocides.Disc diffusion assays were used to determine the efficacy of the biocides benzalkonium chloride, sodium hypochlorite, and 4-chloro-3,5-dimethylphenol on predominant isolates.4-chloro-3,5dimethylphenol and benzalkonium chloride demonstrated significantly greater antimicrobial activity than sodium hypochlorite. Concentration was a significant factor affecting growth inhibition only for benzalkonium chloride.

Keywords: Biocides, shared kitchens, 4-chloro-3,5-dimethylphenol, benzalkonium chloride, sodium hypochlorite

(c) RASĀYAN. All rights reserved
\end{abstract}

\section{INTRODUCTION}

The domestic kitchen is increasingly being recognized as the most important area in relation to the incidence of foodborne disease. Effective biocide use in the kitchen is essential in order to minimize the risk of infection. Furthermore, few kitchen studies such as that by Scott et al. ${ }^{1}$ have investigated the susceptibility of predominant kitchen isolates to relevant and commonly used biocides. Chemical agents with antiseptic, disinfectant and/or preservative activity fall under the umbrella term 'biocides'. ${ }^{2}$ Biocides are defined as "molecules, generally of synthetic or semisynthetic origin, that, above certain critical concentrations and under defined conditions, will kill living cells within specified times". ${ }^{3}$ It is important to recognize the difference between antibiotics and biocides. Antibiotics work in conjunction with host defense mechanisms to eliminate bacteria, generally acting on a single target to achieve growth inhibition. ${ }^{4}$ Conversely, biocides may act on one or multiple cellular targets with the aim of rapid killing. Broad classifications such as oxidative, membrane active etc. have been assigned to biocides as a result. ${ }^{3}$ Potential bacterial target sites for biocides include the cell wall or outer membrane, cytoplasmic membrane, DNA and RNA amongst other cytosolic components. ${ }^{5}$ Factors that may influence biocide activity include concentration, period of contact, $\mathrm{pH}$, temperature, presence of organic matter and the microorganism's nature, number, location and condition. ${ }^{2}$ It is worthy to note that there is currently a severe lack of evidence to suggest that the exposure of bacterial cultures to sublethal concentrations of biocide is related to the emergence of antibiotic resistance. ${ }^{3}$ The biocides used in this study are: sodium hypochlorite, 4-chloro-3,5-dimethylphenol (also known as chloroxylenol) and benzalkonium chloride. They were selected for use in this study due to their presence in a wide range of branded domestic kitchen cleaners such as Clorox, Dettol and Cif respectively. The aim of this study is to assess the susceptibility of microorganisms isolated from a shared student kitchen to sodium hypochlorite, 4-chloro-3,5-dimethylphenol (chloroxylenol) and benzalkonium chloride.

\section{EXPERIMENTAL}

\section{Isolates used in the study}

D1: Staphylococcus spp./Micrococcus spp., D2: Enterobacteriaceae,

D3: Pseudomonas aeruginosa, D4: Pseudomonas spp., D5: Enterobacteriaceae, 
S1: Pseudomonas spp., S2: Pseudomonas spp., S3: Enterobacteriaceae,

S4: Staphylococcus aureus, S5: Pseudomonas spp., R1: Pseudomonas spp.,

R2: Enterobacteriaceae, R3: Pseudomonas spp., R4: Bacillus spp., and

R5: Pseudomonas spp.,

\section{Preparation of disc diffusion assay plates}

Each bacterial culture was spread onto 5 nutrient agar plates; one for each antimicrobial, one for a positive control and one for a $96 \% \mathrm{v} / \mathrm{v}$ ethanol control as the 4-chloro-3,5-dimethylphenol was dissolved in ethanol. The plates were then prepared using the three antimicrobials. The ethanol control was prepared in the same way but using only two discs instead of four as only one concentration was under investigation. All plates were then incubated overnight at $37^{\circ} \mathrm{C}$.

\section{Zones of inhibition}

The diameters of all observed zones of inhibition were measured using a ruler and recorded. Any merged zones were also noted.

\section{RESULTS AND DISCUSSION}

\section{Susceptibility of predominant isolates to selected kitchen biocides (Kirby-Bauer method)}

The results from the disc diffusion assays carried out on each predominant isolate are presented in Fig.- 1 . The $96 \% \mathrm{v} / \mathrm{v}$ ethanol control experiments revealed statistically insignificant $(\mathrm{P}>0.05)$ bactericidal activity on the predominant isolates. The significance of the difference in mean inhibition zones between the different biocides was calculated using an unpaired student T-test. A paired student T-test was used to calculate the significance of this difference for different concentrations of the same biocide. A comparison of the mean inhibition zone diameter created by each concentration of biocide is illustrated in Fig.- 2 .

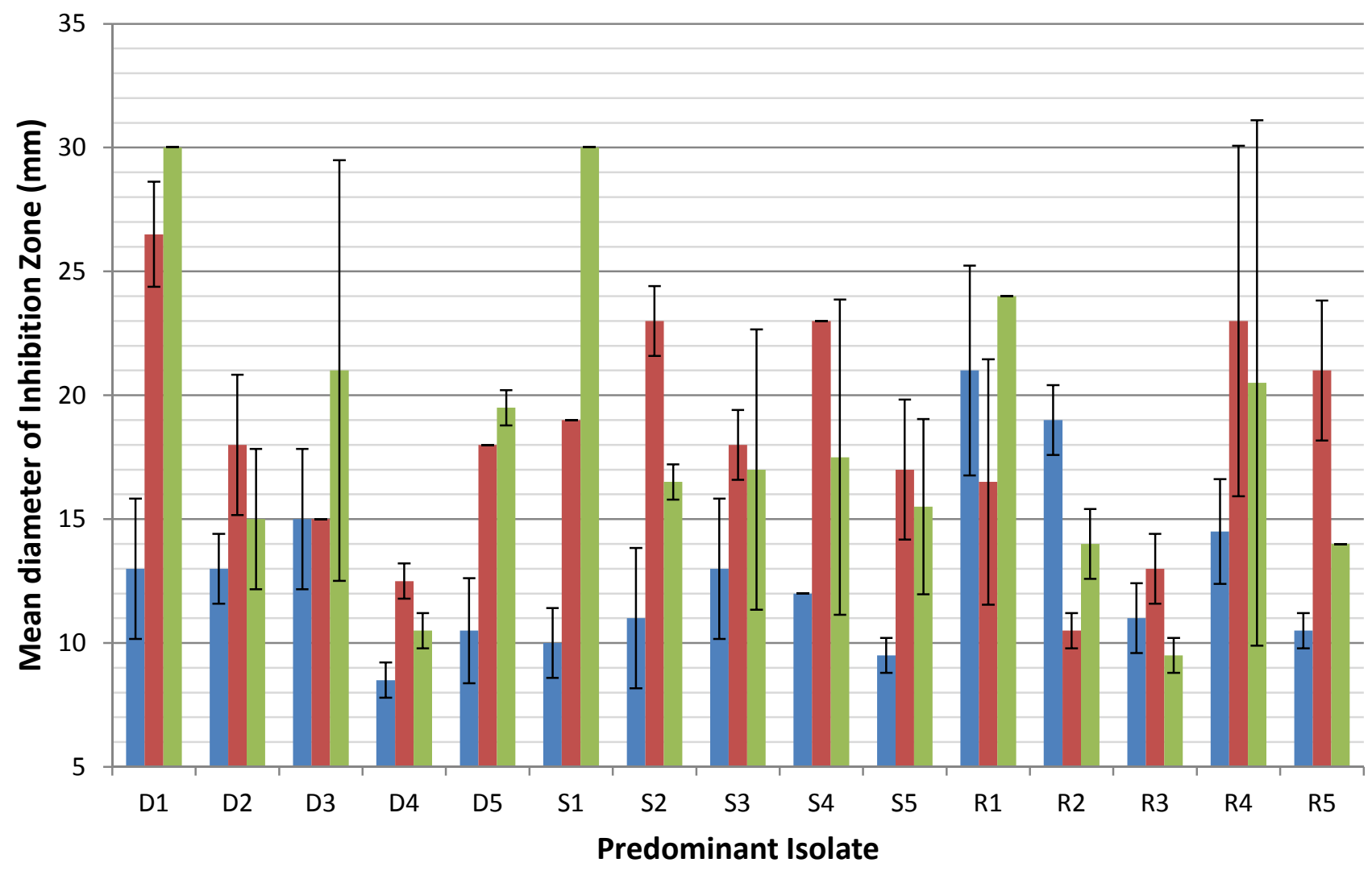

$\square$ 2\% Sodium Hypochlorite $\quad$ 1\% Benzalkonium Chloride $\quad 1 \%$ 4-Chloro-3,5-dimethylphenol 
RASĀYAN J. Chem.

Vol. 11 | No. 1 |238-244 | January - March | 2018

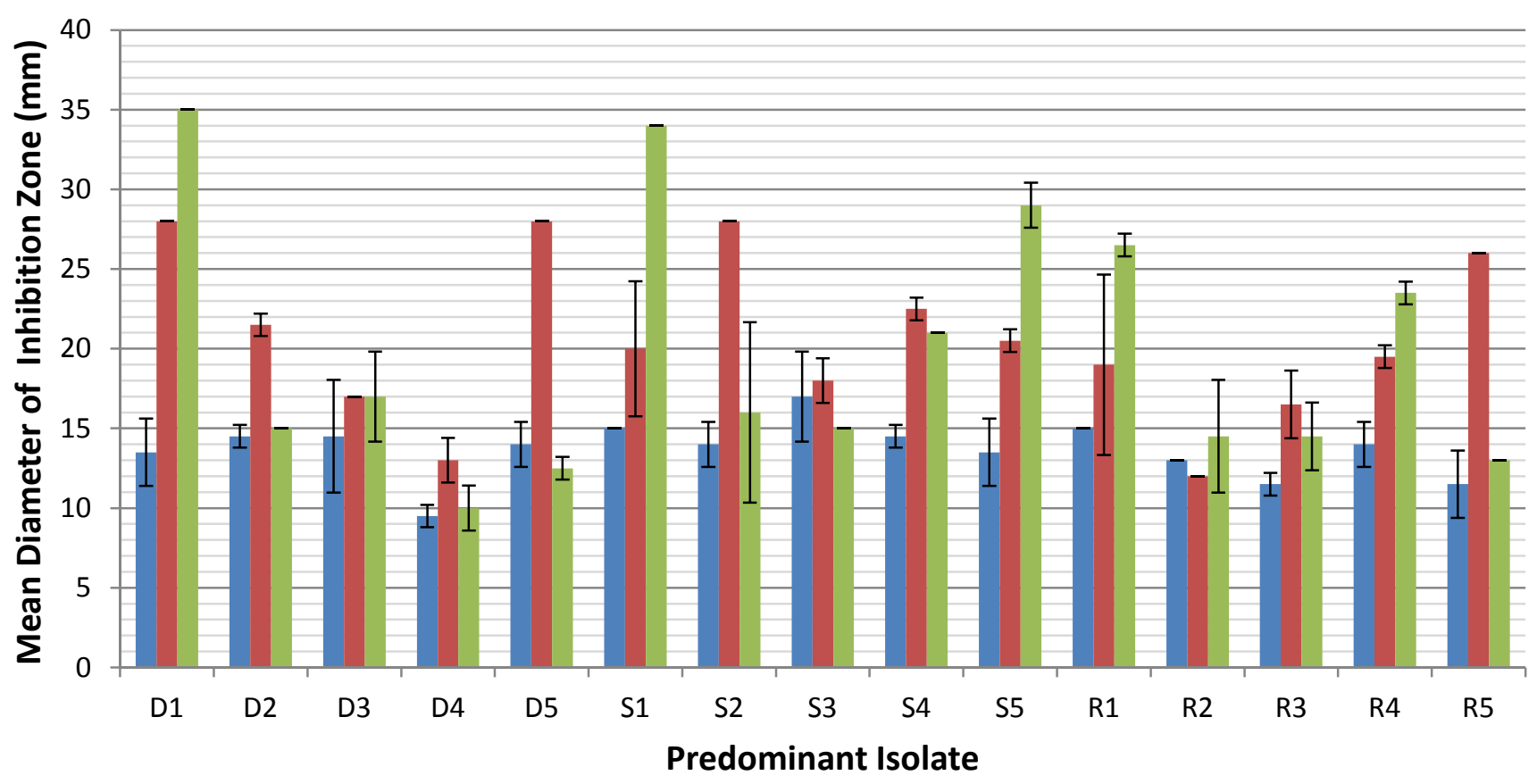

घ\% Sodium Hypochlorite $\quad$ 2\% Benzalkonium Chloride $\quad 2 \%$ 4-Chloro-3,5-dimethylphenol

Fig.-1: Graphs showing the mean inhibition zone diameters observed when the predominant isolates were treated with low (Fig.-1a) and high (Fig.-1b) concentrations of three biocides commonly used in the kitchen. The data are means from duplicate experiments \pm standard deviation. Larger inhibition zone diameters reflect greater susceptibility of an isolate to the biocide.

\section{Comparison of the different biocides}

Benzalkonium chloride resulted in significantly larger mean inhibition zones $(\mathrm{P}<0.05)$ than sodium hypochlorite when both, high and low, concentrations were compared. The same was found to be true when 4-chloro-3,5-dimethylphenol was compared to sodium hypochlorite. However, the difference in mean inhibition zones between benzalkonium chloride and 4-chloro-3,5-dimethylphenol was found not to be statistically significant $(\mathrm{P}>0.05)$ when the same concentrations of each were compared.

\section{Comparison of the different concentrations of each biocide}

$2 \%$ benzalkonium chloride resulted in significantly larger mean inhibition zones $(\mathrm{P}<0.05)$ than $1 \%$ benzalkonium chloride. However, the difference in mean inhibition zones observed for different concentrations of sodium hypochlorite and 4-chloro-3,5-dimethylphenol was found not to be statistically significant $(\mathrm{P}>0.05)$.

\section{Susceptibility of the predominant isolates}

The predominant isolates D4 and R3, both presumptively identified as Pseudomonas spp., showed relatively low susceptibility to treatment with all three biocides irrespective of concentration used. Isolates D1 and S1, presumptively identified as Staphylococcus spp. or Micrococcus spp. and Pseudomonas spp. respectively, showed relatively high susceptibility to treatment with both concentrations of all three biocides. Staphylococcal isolates appear to be less susceptible to sodium hypochlorite relative to the other two biocides tested irrespective of the concentration used. It would also appear that Gram-positive species were generally more susceptible to benzalkonium chloride than Gram-negative species.

Very little published information relating to the use of disinfectants in non-healthcare settings ${ }^{6}$ such as the domestic kitchen prompted the final objective of this study: to assess the susceptibility of the predominant 
kitchen isolates to three relevant and commonly used kitchen biocides. Susceptibility to two different concentrations of each of sodium hypochlorite, chloroxylenol (4-chloro-3,5-dimethylphenol) and benzalkonium chloride was investigated using the agar disc diffusion method (Kirby-Bauer method).

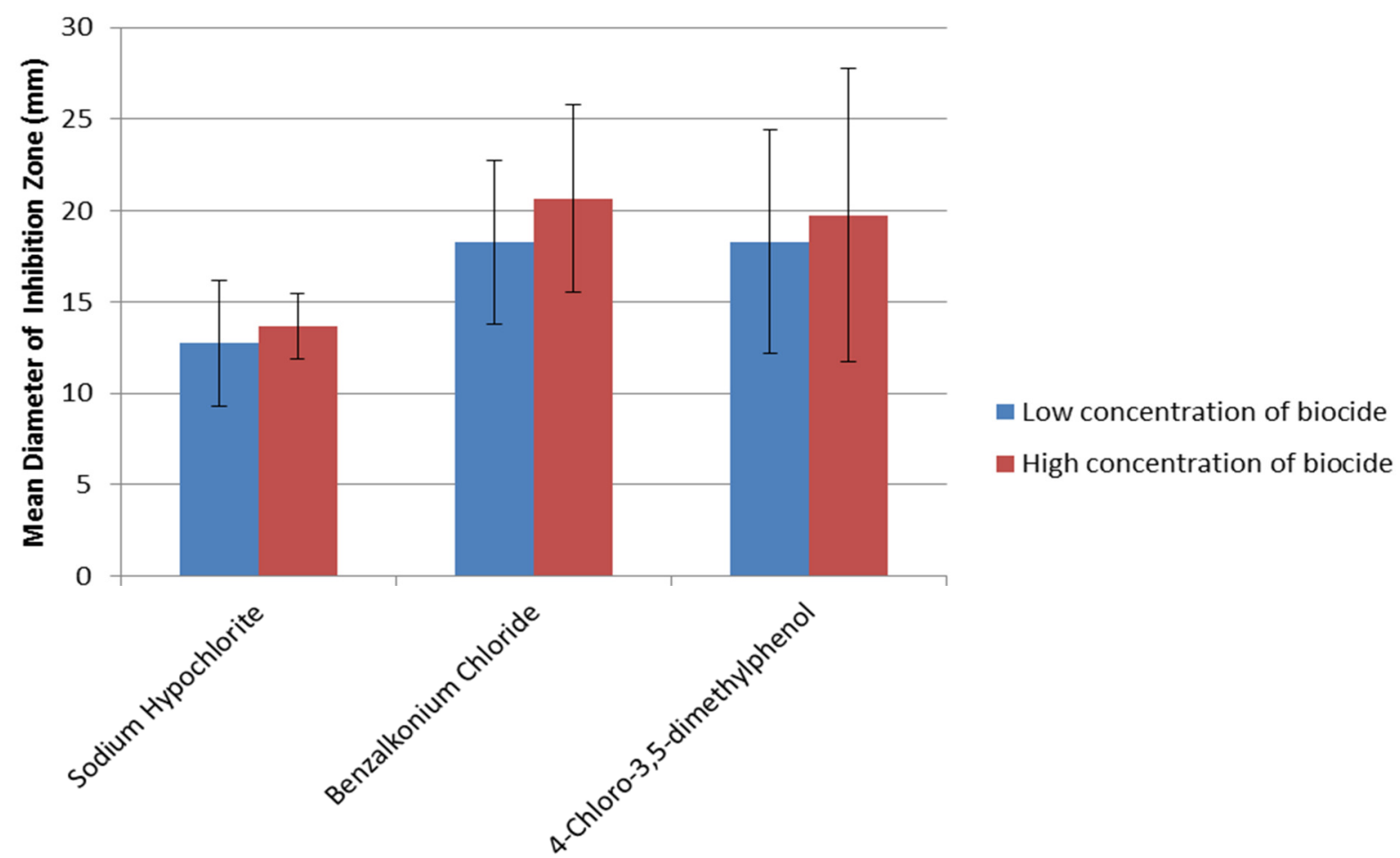

Fig.-2: Graph showing the mean inhibition zone diameter produced by each concentration of biocide. The data are means of all predominant isolates treated \pm standard deviation. A larger mean inhibition zone diameter reflects the greater antimicrobial activity of the biocide. Low concentration of biocide: $2 \%$ sodium hypochlorite, $1 \%$

benzalkonium chloride, 1\% 4-chloro-3,5-dimethylphenol; high concentration of biocide: 3\% sodium hypochlorite, $2 \%$ benzalkonium chloride, $2 \%$ 4-chloro-3,5-dimethylphenol.

The agar disc diffusion tests revealed that benzalkonium chloride and 4-chloro-3,5-dimethylphenol (chloroxylenol) were both generally more effective than sodium hypochlorite irrespective of concentrations used, resulting in significantly greater growth inhibition i.e. larger mean inhibition zone sizes. The concentration used was found to significantly affect growth inhibition only in the case of benzalkonium chloride where a concentration of $2 \%$ produced significantly larger mean inhibition zones than $1 \%$. The effect of concentration using 4-chloro-3,5-dimethylphenol and sodium hypochlorite on growth inhibition was found to be statistically insignificant. It is important to note that in no cases did any of the three biocides tested fail to produce a zone of inhibition. This shows that all three biocides tested exhibited antimicrobial activity against all the microorganisms isolated from the kitchen in this study, albeit to varying degrees.

While no definite conclusions can be made regarding genus type and susceptibility, due to the many factors affecting the accuracy of the disc diffusion method discussed later in this report, it would appear that the staphylococcal isolates (D1 and S4) were less susceptible to sodium hypochlorite relative to the other two disinfectants tested irrespective of the concentration used (2\% and 3\%); this is illustrated in Figures 1a and $1 \mathrm{~b}$. This finding is supported by a study by Kusumaningrum et al. ${ }^{7}$ who found that Staphylococcus aureus exhibited better tolerance to sodium hypochlorite relative to Salmonella enteritidis, with only small zones of inhibition observed using the agar diffusion test.

It would also appear that the Gram-positive species tested in this study were more sensitive to benzalkonium chloride than Gram-negatives, with generally larger inhibition zones observed with the former. This finding concurs with Tebbs and Elliot ${ }^{8}$ who observed larger inhibition zones with Gram-positive staphylococcal species than with Gram-negative species reporting that benzalkonium chloride may require a prolonged 
contact time with the latter to be as effective. This is most likely explained by the presence of an outer membrane unique to Gram-negative bacteria which may act as a barrier ${ }^{9}$ prolonging the time to penetration of benzalkonium chloride.

At present, the underlying causes of the varied susceptibilities of different microorganisms to biocides are poorly understood but are thought to include differences in the structure of outer cell layers affecting the absorption of biocides into cells, differences in the affinity and amounts of target site(s); differences in stress responses; and growth within a biofilm. ${ }^{9}$

However, in addition to varied susceptibilities of the isolates ${ }^{8}$, the varying zone sizes observed with the different biocides can be attributed to their different size (molecular weight) and shape, charge, and concentration in the agar medium which all have an effect on the rate of diffusion through the medium and thus on the extent of growth inhibition observed. ${ }^{10}$ Additionally, the volume of agar poured into each plate and hence the depth of the agar in the plate also affects the rate of diffusion, with relatively less antimicrobial available to diffuse through excessively thick layers resulting in falsely small zones of inhibition and vice versa. Furthermore, the stacking of plates in the incubator has been shown to cause a delay in reaching the optimum temperature for growth and multiplication of the test bacteria resulting in slightly larger zones being observed on plates in the center relative to those at the top or bottom of the pile. . $^{10,11}$

Although direct comparison with other studies is difficult due to variations in test methods ${ }^{12}$ and concentrations of biocide used ${ }^{13}$, the results relating to sodium hypochlorite appear contrary to what some other studies have reported. ${ }^{1,14-17}$ Siqueira $\mathrm{Jr}$ et al. ${ }^{16}$ reported large zones of inhibition, ranging from $18.5 \mathrm{~mm}$ to $31 \mathrm{~mm}$, when sodium hypochlorite concentrations of $1 \%, 2.5 \%$, and $5.25 \%$ were tested against Enterococcus faecalis by means of agar diffusion and found that the higher concentrations yielded significantly larger zones. Sassone et al. ${ }^{17}$ also observed significant zones of inhibition when $1 \%$ and 5\% concentrations of sodium hypochlorite were tested against a range of bacteria, including Enterococcus faecalis, E. coli and S. aureus, stating that significantly larger inhibition halos were observed with the higher concentration. However, interestingly, when the agar diffusion tests were repeated in the presence of organic material, no inhibition zones were observed with either concentration leading the authors to conclude that organic load interferes with the biocide's antimicrobial activity in agar diffusion tests. The same conclusion was also reached in a study by Madrid and colleagues. ${ }^{18}$ This was attributed to a reaction of the biocide with the organic material.

Since how well an antimicrobial diffuses through the agar medium is critical and proportional to zone size observed $^{7,10,11}$, reactions with organic materials (protein components) ${ }^{17,18}$ in the nutrient agar may help explain why sodium hypochlorite produced relatively small zones in this study (as shown in Figures 1a and 1b) compared to the above studies which utilized different agar types. Binding to organic/protein components has also been shown to be an issue affecting the antimicrobial activity of quaternary ammonium compounds (QACs) such as benzalkonium chloride. ${ }^{7,8}$

Another explanation may involve the dependence of the antimicrobial effectiveness of sodium hypochlorite on $\mathrm{pH} .{ }^{18,19}$ Madrid et al. ${ }^{18}$ stated that more alkaline $\mathrm{pH}$ results in longer stability of hypochlorite solutions. Therefore the acid $\mathrm{pH}$ of the nutrient agar used in this study may have resulted in instability of the biocide and hence a negative effect on its antimicrobial efficacy.

Scott et al. ${ }^{1}$ investigated various disinfection and cleaning procedures in an 'in home' study concentrating on 'wet sites' most likely to harbor potentially infectious pathogens, such as the kitchen sink surface, Utube and draining board mentioned previously. The authors used phenolic and hypochlorite disinfectants and found that, although both resulted in rapid significant decreases in the levels of contamination, especially in the incidence of Enterobacteriaceae; the latter was more effective. However, they also warned that it would be inappropriate to make the generalization that hypochlorite disinfectants were always more effective than phenolics due to considerable variations in strengths of commercially available bleach products, pointing out that 'in use' concentrations can significantly vary from the concentrations used in their study. Likewise, such generalizations should be avoided when interpreting the data in this study as the sodium hypochlorite concentrations used ( $2 \%$ and $3 \%$ ) were lower than the up to $15 \%$ concentrations found in commercial products for home or hospital use. ${ }^{20}$ 
As Johnson et al. ${ }^{12}$ correctly noted, there is very little data regarding bacterial susceptibility to chloroxylenol in the literature and interpretation of this data is further complicated by varying test methods. The authors found that susceptibility to chloroxylenol varied between strains and species which correlates with the findings in this study. However, more specific correlations are difficult to make at this time. Unsurprisingly, they also found that biofilm-grown cells were two-to eightfold less susceptible than their planktonic counterparts irrespective of strain. This highlights the importance of additionally carrying out susceptibility tests on microorganisms growing on a surface in order to obtain a more accurate indication of the 'in use' antimicrobial activity of biocides.

Only a few variables affecting inhibition zone sizes in the agar disc diffusion method have been discussed above, further variables have been identified and discussed in detail by Tobias. ${ }^{10}$ Due to the several variables associated with this method, it can be difficult and time-consuming to carefully control each of them in order to achieve consistent and reproducible results. ${ }^{10}$ Furthermore, while this method does provide an indication of whether or not a microorganism is susceptible to the biocide tested, it does not distinguish between bacteriostatic and bactericidal properties of the test material nor does it allow for conclusions regarding the viability of test microorganisms to be made. ${ }^{10}$ It is also important to note that biocides, and especially disinfectants, used in the domestic kitchen are preferentially required to kill bacteria making tests such as the agar diffusion method, which measures the growth-inhibitory ability of a substance, insufficient for this purpose, providing only preliminary information. ${ }^{21}$

Suspension and surface disinfection tests may perhaps be better alternatives for evaluating antimicrobial activity more thoroughly for the purposes of this study, with the latter being more superior due to more closely representing the 'in practice' use of biocides in the domestic kitchen. ${ }^{21}$

Suspension tests involve adding a specified amount of test bacteria suspension to a prepared volume of the biocide being investigated for a specified contact time after which an aliquot is removed, neutralized to quench biocide activity, and plated out using standard culture techniques (e.g. drop counting method) in order to determine the number of survivors and thus allow for the reduction in viable count to be calculated. In contrast, in surface disinfection tests the test bacteria is dried onto the surface of a specified carrier and the biocide under investigation is added over the top of the dried cells for a specified contact time. The cells are then neutralized, re-suspended and the reduction in viable count calculated using the same method described for suspension tests. It is widely acknowledged that bacteria in the environment preferentially grow in association with a surface forming biofilms, thus the latter method would allow for a more accurate indication of how a biocide would perform in practice. Furthermore, Thomas et al. ${ }^{21}$ demonstrated that cells dried onto a surface were less susceptible to chlorhexidine and benzalkonium chloride than cells in suspension. This demonstrates the relatively increased recalcitrance of surface-associated bacteria and the importance of carrying out surface disinfection tests when evaluating the antimicrobial activity of biocides, particularly those intended for use in the kitchen environment.

\section{REFERENCES}

1. E. Scott, S. F. Bloomfield and C. G. Barlow, The Journal of Hygiene, 92, 2, 193(1984)

2. A. D. Russell, The Lancet Infectious Diseases, 3, 12, 794(2003)

3. P. Gilbert and A. J. Mcbain, Clinical Microbiology Reviews, 16, 2, 189(2003)

4. S. F. Bloomfield, Journal of Applied Microbiology, 92, 144S(2002)

5. A. Bridier, R. Briandet, V. Thomas and F. Dubois-Brissonnet, Biofouling, 27, 9, 1017(2011)

6. W. A. Rutala, S. L. Barbee, N. C. Aguiar, M. D. Sobsey and D. J. Weber, Infection Control and Hospital Epidemiology, 21, 1, 33(2000)

7. H. D. Kusumaningrum, R. Paltinaite, A. J. Koomen, W. C. Hazeleger, F. M. Rombouts and R. R. Beumer, Journal of Food Protection, 66, 12, 2289(2003)

8. S. E. Tebbs and T. S. Elliott, The Journal of Antimicrobial Chemotherapy, 31, 2, 261(1993)

9. A. D. Russell, Journal of Antimicrobial Chemotherapy, 52, 5, 750(2003)

10. R. S. Tobias, International Endodontic Journal, 21, 2, 155(1988)

11. W. W. Davis and T. R. Stout, Applied Microbiology, 22, 4, 659(1971) 


\section{RASĀYAN J. Chem.}

Vol. 11 | No. 1 |238-244 | January - March | 2018

12. S. A. Johnson, P. A. Goddard, C. Iliffe, B. Timmins, A. H. Rickard, G. Robson and P. S. Handley, Journal of Applied Microbiology, 93, 2, 336(2002)

13. A. D. Russell and G. Mcdonnell, Journal of Hospital Infection, 44, 1, 1(2000)

14. E. Scott and S. F. Bloomfield, Journal of Applied Microbiology, 68, 3, 279(1990)

15. P. Rusin, P. Orosz-Coughlin and C. Gerba, Journal of Applied Microbiology, 85, 5, 819(1998)

16. J. F. Siqueira Jr, I. N. Rôças, A. Favieri and K. C. Lima, Journal of Endodontics, 26, 6, 331-334 (2000)

17. L. M. Sassone, R. A. Fidel, C. F. Murad, S. R. Fidel and J. R. Hirata, Australian Endodontic Journal, 34, 1, 19(2008)

18. I. M. Madrid, A. S. Mattei, R. Santin, A. Dos Reis Gomes, M. B. Cleff and M. C. Meireles, Mycoses, $\mathbf{5 5}, 3,281(2011)$

19. M. Mercade, F. Duran-Sindreu, S. Kuttler, M. Roig and N. Durany, Oral Surgery, Oral Medicine, Oral Pathology, Oral Radiology, and Endodontology, 107, 2, 295(2009)

20. W. A. Rutala and D. J. Weber, Clinical Microbiology Reviews, 10, 4, 597(1997)

21. L. Thomas, A. D. Russell and J. Y. Maillard, Journal of Applied Microbiology, 98, 3, 533(2005)

[RJC-2019/2017] 